Original article

\title{
The clinical reasoning processes of extended scope physiotherapists assessing patients with low back pain
}

\author{
Neil Langridge*, Lisa Roberts, Catherine Pope \\ University of Southampton, Faculty of Health Sciences, Building 67, Highfield, Southampton, SO17 1BJ, UK
}

\section{A R T I C L E I N F O}

\section{Article history:}

Received 14 July 2014

Received in revised form

11 January 2015

Accepted 15 January 2015

\section{Keywords:}

Physiotherapy

Reasoning

Back

Pain

\begin{abstract}
A B S T R A C T
Introduction: Employing allied health professionals in extended scope roles has developed relatively recently in health-care. Within physiotherapy, the extended role has provided clinicians with autonomy to use knowledge and clinical acumen to request investigations such as Magnetic Resonance Imaging (MRI) and X-ray as part of the diagnostic process, a practice beyond the traditional scope of physiotherapy. In these advancing roles, little is written about the clinical reasoning processes that clinicians use in managing patients with musculoskeletal pain and knowledge of these processes would advance training for new recruits to this arena.

Study: This qualitative study has explored the processes by which extended scope physiotherapists (ESPs) clinically reason their decisions regarding patients reporting low back pain in a musculoskeletal outpatient setting. The study used a multiple case study design informed by grounded theory methodology, using focus groups (involving ESPs and non-ESPs/musculoskeletal physiotherapists) and semistructured interviews with a think-aloud method (ESPs only) to investigate these processes.

Conclusions: The themes identified include: prior thinking; patient interaction; formal testing; time; safety and accountability; external and internal factors; and gut-feeling (which challenges current physiotherapy models of reasoning). Extended scope physiotherapists reported experiencing greater stress due to higher levels of perceived accountability, safety requirements and internal drivers for competence than non-ESPs. Further research is indicated to explore the role of gut-feeling in musculoskeletal physiotherapy clinical reasoning.
\end{abstract}

๑) 2015 Elsevier Ltd. All rights reserved.

\section{Introduction}

Clinical reasoning refers to the thinking and decision-making processes that are used in clinical practice (Edwards et al., 2004). It is regarded an integral, vital component to being a clinician (Norman, 2005), and is a skill that begins in training and is refined with experience (Doody and McAteer, 2002; Curran et al., 2006; May et al., 2008).

Forde (1998) describes clinical reasoning as a continuum; at one end of the continuum is the strongly-embedded scientific, analytical approach that entails hypothesis-testing or systematic sorting of clinical data (Jefford et al., 2010), while at the opposite end lies a more intuitive process that does not resemble these 'scientific' methods.

\footnotetext{
* Corresponding author. Lymington New Forest Hospital, Wellworthy Road, Lymington, Hants, SO41 8QD, UK. Tel.: +4401590 663263; fax: +44 01590663264.

E-mail address: neil.langridge@southernhealth.nhs.uk (N. Langridge).
}

This paper explores clinical reasoning by drawing on an empirical study of physiotherapists to understand which models of reasoning best explain practice. The study reported here explored whether extended scope practitioners draw on different reasoning skills in the assessment of low back pain, and it sought to inform future training and current practice.

\subsection{Clinical reasoning models in physiotherapy}

Within musculoskeletal practice, four commonly cited models of reasoning are hypothetico-deductive, pattern recognition, narrative and clinical prediction (Flynn et al., 2002; Edwards et al., 2004). These models are also established and accepted within other healthcare professions (Higgs and Jones, 2008).

The hypothetico-deductive model remains the most enduring clinical reasoning approach in medicine, and early studies involving physiotherapists also suggested that reasoning progresses from a "diagnosis" or hypothesis proposing 'the problem' followed by 
testing to rule out different answers (Payton, 1985). Pattern recognition requires the clinician to make quick assumptions based on memory and experience (Patel et al., 1997). This model has developed from cognitive psychology and proposes that clinicians use "illness scripts", which are clusters of presentations that are supported by previous experience (Arocha et al., 1993). Narrative reasoning aims to establish insight into the patients' perspective and story, rather than testing for "cause and effect" (Edwards et al., 2004; Banning, 2008). The final model, clinical prediction, describes a reasoning process that identifies common variables within a patient presentation that linked together suggest a successful treatment programme (Flynn et al., 2002).

\subsection{Study context}

Since 1986, the role of physiotherapists has been extended in response to the need to reduce costs and waiting times for patients (Daker-White et al., 1999). Extended scope physiotherapists (ESPs) work within a range of services crossing established boundaries of practice between medicine, nursing and allied health professionals (Gardiner and Turner, 2002; McPherson et al 2006). Enhancing pathways to appropriate management has been a key UK government directive (DOH, 2006; 2013) and in response many services have looked at innovative ways to deliver care, such as providing extended roles, which in turn has fuelled the growth of ESP practice.

Advanced/ESP practitioners are expected to combine clinical reasoning and reflection (Dewar, 2010). Understanding how these clinicians formulate decisions is especially important because they represent an increasingly autonomous profession (Higgs, 1999). The skills demonstrated by ESPs are different from non-ESPs, for example, they may include a triage role, capacity to order investigations, to perform non-physiotherapy procedures such as injections, and refer on to medical and surgical services (Dawson and Ghazi, 2004).

To capture clinical reasoning practices, the research presented here adopted an interpretive approach which sought to understand the narrative, contextual and interpersonal components of clinical decision-making.

\section{Methodology and methods}

Ethical approval was granted by the Southampton and South West Hampshire Research Ethics Committee (10/HO504/3 phase 1, 11/SC/008 phase 2).

A case study design, informed by grounded theory, was chosen to address the research question "What are the clinical reasoning processes of extended scope physiotherapists assessing low back pain?".

A case study focuses on a single entity, or a phenomenon which has boundaries that allows it to be explored in detail (Miles and Huberman, 1994). This study compared the clinical reasoning processes of ESPs to non-ESPs to develop an understanding of the reasoning processes they used, followed by further analysis of ESP reasoning, using a different method. The study had a two-stage design. Initially, three focus groups were completed, involving both ESPs and non-ESPs in each group, to identify the reasoning processes they used and the differences between the ESPs and nonESPs. Data from these focus groups informed a second stage of data collection, whereby semi-structured interviews were conducted with ESPs directly after a patient consultation. This method used in this phase was "think-aloud", a qualitative tool used to analyse problem-solving (MacNeela et al., 2010) which aimed to help further interpret the specific reasoning of ESPs in the assessment of patients reporting back pain.

\subsection{Participants}

For the focus groups, ESPs and non-ESPs were purposively selected at three NHS Trusts located in England. Primary and secondary care services were included to enhance applicability of the findings to the range of services where physiotherapists practice. Each focus group involved six participants, three ESPs and three non-ESPs. The second phase of the study was expanded to cover four further NHS Trusts, to include participants who were not known to the researcher, in an attempt to reduce potential bias in these data. Ten ESPs were recruited for phase two of the study.

\subsection{Consent}

Managers of musculoskeletal services were approached for the focus group study and were asked to deliver information sheets and consent forms to potential participants. For phase two, managers of ESP spinal clinics were approached in the same way. Participants contacted the lead author and the focus groups/interviews were arranged at a convenient time. Written consent was obtained prior to the focus group or interview.

Participants were included if they were members of the Chartered Society of Physiotherapy, registered with the Health and Care Professions Council and working clinically in the National Health Service (NHS). Physiotherapists undergoing ESP training, and therefore working under supervision, were excluded from both phases of this study.

\subsection{Data collection}

Phase 1: The focus groups were facilitated by the lead author and observed by an independent researcher (who recorded her observations). The focus groups used a topic-guide comprising of open questions covering diagnosis; physical testing and clinical reasoning. They were audio-recorded and transcribed verbatim. Each focus group lasted approximately $40 \mathrm{~min}$. Participants were not given transcripts of the focus group data.

Phase 2: Interviews were scheduled immediately after a participating ESP had completed a clinical assessment with a patient in clinic (without the researcher being present). The interview involved reflection on the assessment they had just completed and occurred in the clinic room without the patient present and was audio-recorded. The clinicians had access to their clinical notes from the patient assessment and were asked to talk through their reasoning following the think-aloud process. These interviews lasted approximately $45 \mathrm{~min}$ and were transcribed verbatim. Additional field notes (taken by the lead author) were used to clarify the context of the discussion.

\subsection{Analysis}

Analysis of the data was guided by the principles of grounded theory (Glazer and Strauss, 1967; Strauss and Corbin, 1990; Charmaz, 2008). Three phases of coding were completed: i) line by line transcripts were read for an initial overview and each line was then systematically coded and memos identifying the researchers' initial thoughts were made; ii) axial coding, which involved linking and grouping codes from the different data sources; iii) selective coding, which further grouped these codes, identifying and linking to the main emerging themes (Charmaz, 2008) to produce a theoretical framework. The constant comparison method was used to compare ESP and non-ESP accounts, looking for similarities and differences and highlighting deviant cases. This work was underpinned by regular supervisory review of the emerging interpretations and a process of reflexivity that considered the impact of the researcher upon the data collection and analysis. 


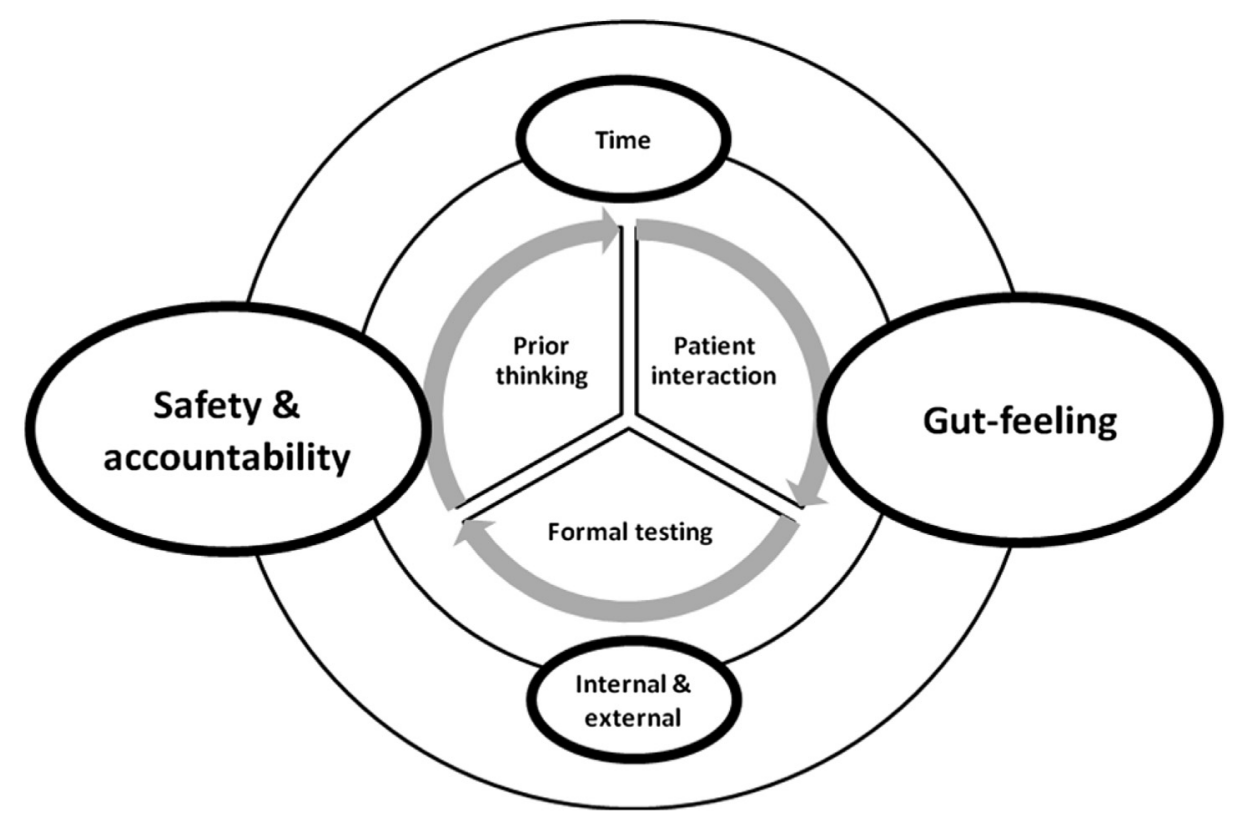

Fig. 1. Model of ESP clinical reasoning in the assessment of patients with LBP.

\section{Findings}

\subsection{Demographics - phase 1 and phase 2}

In phase one of the study (focus groups), 18 physiotherapists participated: 5 male and 4 female non-ESPs, with an equivalent number and gender ratio of ESPs. The average years qualified of the male non-ESPs was 5.8 (range 5-8), female non-ESPs 10.75 (6-13), male ESPs 14.2 (10-16) and female ESPs 21.2 (14-28). The female ESPs had been in post for an average of 4.8 years (3-10) compared to the males at $6.25(5-7)$ years. In phase two, 4 male and 6 female ESPs were interviewed, 4 working in secondary care, 6 in primary care. The average years qualified as a physiotherapist was 17.1 years $(11-22)$ and in post as an ESP, 5.6 years (2-9). Patients in the clinics were all reporting LBP and associated symptoms such as sciatica and were referred by GPs or physiotherapy colleagues.

The main themes are described below and two pictorial models are developed to explain the inter-relationships between the themes and differences in reasoning processes. The models illustrate an inner cyclical process and two outer influential circles which house the further themes. The models demonstrate a process of gaining information via prior thinking, patient interaction and formal tests. In both ESP and non-ESP reasoning these processes occurred in a similar fashion although as discussed below, subtle differences were noted. The models highlight in particular how 'gut feeling' and "safety and accountability" had a greater influence on ESP reasoning and the larger sections in the model reflect this. The non-ESPs did not highlight specific areas that out-

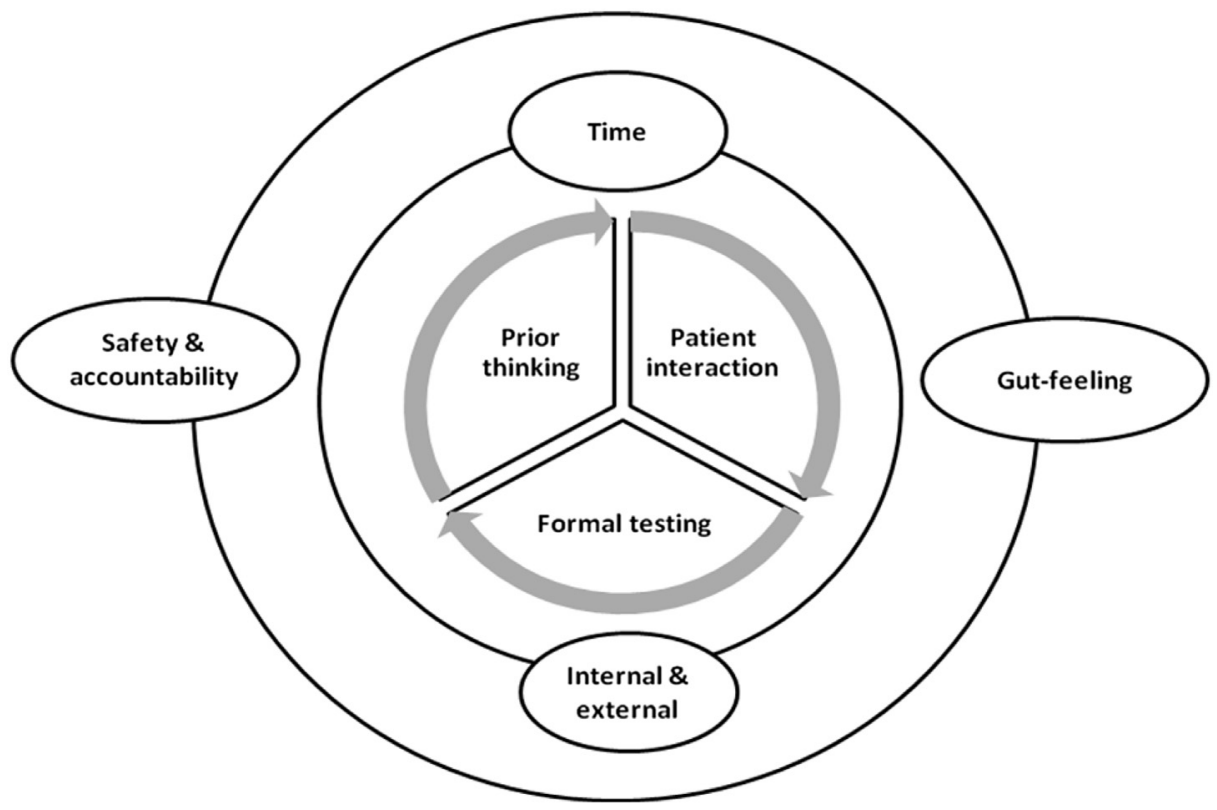

Fig. 2. Model of non-ESP clinical reasoning in the assessment of patients with LBP. 
weighed others in terms of importance, and so are shown here as fairly equal in standing, whilst the outer circle in the ESP model shows themes with identified greater influence upon the decisionmaking process and subsequently underpinned much of the reasoning in this clinical group. Figs. 1 and 2.

\subsection{Theme 1: prior thinking}

This theme is defined as the use of knowledge gained about the patient or their presenting condition prior to a consultation and comprises two elements.

1. Natural knowledge. This is an understanding and appreciation of natural aetiology and biological processes.

2. Clinical patterns. This is a form of pattern recognition drawing on memories of clinical signs and symptoms that have been encountered before in similar cases.

Prior thinking is a way of linking the 'expected' to the 'reality' with knowledge and pattern recognition working together to inform the direction and aims of the assessment (i.e. searching for pathology or finding a movement problem). One ESP described the process as drawing on knowledge (clinical impression) with 'feeling' for a relevant pattern (pain mechanism) thus:

"I think working in an ESP clinic could be that my hypotheses, my clinical impression of that patient would start with potential pathology, you know, get a feeling for what I feel is the dominant pain mechanism." FG 1 ESP 642-644

In contrast a non-ESP suggested looking for patterns while assessing movement:

"I might try and do some of my combined movements looking for an opening pattern, moving pattern” FG 2 Non-ESP 278

In both cases the clinician compares their perceived knowledge (what they think they know about a condition) and expectations to what they actually find out about the patient.

\subsection{Theme 2: patient interaction}

This theme encompasses the clinical relationship and mutual understanding that engenders patient confidence in the clinician. Confidence in the clinician allows for the delivery of information in a safe environment and can positively improve outcome (Harkapaa et al., 1991; Payton et al., 1998). This involves a narrative reasoning process - listening to the patients' story.

"It could be they've waited 3 months for the appointment so they want to tell you how bad it was and the second time you see them it's actually a lot better and they've unloaded all that." FG 1ESP 456-458

The quote below suggests this listening builds a symbiotic communicative relationship:

"And if you don't listen to them they are hardly likely to listen to you.” FG 2 ESP 201

This clinical rapport (Leach, 2005) appeared to work differently for ESPs and non-ESPs, not least because ESPs often only saw a patient once whereas non-ESPs could build up rapport over several clinical encounters:
"Are we talking about an accumulated rapport or are we talking about a rapport that is established after one assessment... Because you can't compare if you've seen somebody seven times." FG 1 ESP 464-467

Some ESPs felt that rapport in the ESP clinics was vital but others recognised that this was difficult to achieve without several contacts. Rapport seemed to be a variant in the two groups and within the ESP participants. Gaining a rapport very early would seem to be vital in gaining as much from the patient as possible and allowing them to express concerns and worries which would inevitably have a bearing on a successful intervention (Street et al., 2009). The quotes below highlights perceptions surrounding creating a rapport between ESPs and non-ESPs.

"In extended physio I wouldn't have thought they would expect a rapport." FG 1 ESP 470

"The patient already thinks well I'm going to be seen by a specialist, we (non-ESPs) almost have to work harder to get that rapport in the first place" FG 1 non-ESP 416-420

\subsection{Theme 3: formal testing}

This theme concerned the use of physical or non-physical tests that aid in the diagnosis and management of a patient. Here physical tests and diagnostic examinations (such as X-ray) are blended with history-taking and prior thinking. Prior thinking and patient interaction were used to inform the selection of tests. One of the key differences in practice between ESPs and non-ESPs was the ability to order investigations and action the results. This created discussion about whether this is a benefit and whether it has changed their reasoning processes. The ESP clinicians felt that the use of investigations created greater objectivity.

"I suppose you can be slightly more objective in the clinic because you've got the aid of diagnostic tests, so if we made an hypothesis and then had some arranged diagnostic tests, when it comes back its confirming a diagnosis." FG ESP 486-488

"She was very restricted in her walking I felt it was probably ideal to go ahead straightaway and do a MRI scan to actually confirm if it was the case of spinal stenosis." SSI 5 20-25

The ESPs appeared to make more use of diagnostic tests, such as MRI, in contrast to physical tests such as the non-ESPs utilised whose clinical decisions were based on findings from patient examination.

"But I might look for stiffness, such as L5/S1, or explore neural tension, try to put it into sub-headings." FG 1 non-ESP 609-612

This may represent a shift in the way ESPs make clinical decisions, and this could lead to potential conflicts in linking radiological data to the clinical findings as these are not well represented the literature with correlation of radiology and symptoms known to be poor (Videman et al., 2003), for example, in the case of back pain, early use of MRI has been shown to lead to a poorer prognosis, increased chance of disability and greater likelihood of the patient receiving surgery (Graves et al., 2012a, 2012b).

\subsection{Theme 4: safety and accountability}

Safety and accountability incorporates aspects of safe practice, vigilance, and medico-legal issues identified by participants as 
influencing their reasoning. There are three main elements within this theme.

1. Clinician professional safety. This is composed of the clinicians' awareness of their own professional liability.

2. Patient safety. This emphasises perceptions of the clinical safety of the patient which informs decisions.

3. Accountability. This element of the theme relates to perceived accountability and is understood differently by ESP clinicians as the quotes below indicate.

"I am more cautious than I would be in a normal physiotherapy." FG 1 ESP 501

"So that legally it does make a difference and our responsibility is to the patient and not to just dump it (sic) on the physio department" FG 1 ESP 676-677

The pressure in ESP clinics is demonstrated in this theme. Both the ESPs and non-ESPs are accountable to the patient, GP, commissioners, managers and themselves; however the perceived legal levels of accountability are expressed as higher in the ESP group as was their concerns over safety. It is perhaps arguable that this should not be any different in the groups as the patient reporting low back pain may present to either, but it seems the escalation of care to the ESP leaves the ESP feeling greater pressure to not miss anything significant.

\subsection{Theme 5: external and internal factors}

External influences included indirect elements of reasoning processes such as national policy, finance constraints or NHS Trust directives. Internal factors included clinician perceptions of themselves, and awareness of how others view them. Each of these affected their decisions.

"I did work in an environment once where they were actually trying to restrict the number of X-rays, that was not easy to deal with." SSI 5 293-295

"I mean sometimes I feel like I am de-skilling as a physio. I think there can be a lack of appropriate support." SSI 2349

ESPs suggested that internal and external factors negatively impacted upon their clinical decision-making. There was a sense that the ESPs had to justify themselves within a medical environment as well as within a therapeutic one, hence the concern regarding becoming de-skilled. Requesting radiology is expensive and carries risk, the extended role places the ESP in an environment housing greater scrutiny and so places increased pressure upon decision-making.

\subsection{Theme 6: gut-feeling}

Gut-feeling is defined in this research as a sub-conscious process of thought, causing a physical reaction in the clinician which is linked to fear and concern, which in turn generates or supports a clinical decision or action. This was especially important to ESPs

"Gut feeling is perhaps just another term for experience isn't it? I can't remember having too many gut feelings when I started working." FG 2 non-ESP 513-514

For the ESPs, gut-feeling was central to their reasoning processes. For example in drawing on prior thinking, the clinician makes a judgement based on expectation, but for the ESPs this was generated through clinical assumptions, based on limited clinical data available prior to the examination.

"I can't learn gut feeling, so I don't know, I think maybe gut feeling is the things that we identify but aren't conscious that we've identified them maybe." SSI 3 148-151

"There must be a kind of physical reaction or there must be signs in the presentation that are giving us that gut feeling but maybe we just haven't consciously identified what they are." SSI 3 153-155

ESP clinicians described ways in which formal assessment procedures are developed within an automatic/intuitive rather than deliberate, rule-based and analytical process (Bleakley et al., 2003). They then used experiential knowledge to inform the choice of tests to confirm this hunch or intuition.

"We shouldn't really rely on gut feeling, they should be confirmed" Focus group 2 ESP 544-546

This theme played a supportive component in the ESP model. It played a part in how the ESPs considered the patient, the tests and their interpretation, the levels of pressure and safety and helped when time was paramount. Although at times the ESPs were uncomfortable about its relevancy they recognised its use far more than the non-ESPs, but were reticent about it as it seemed far removed from an analytical process, which they felt gave them greater support when justifying their processes within and outside the profession. It seemed to underpin much of what the ESPs did, and was the driver of many cases when a need to make a decision concerned the clinician but they were not clear why. They used this "feeling" to support a decision that they felt was critical, or perhaps needed significant consideration to the presentation.

\subsection{Theme 7: time}

The data concerning time suggested that it had a significant impact on clinical reasoning. A lack of time to build a therapeutic relationship was linked to pressure surrounding the lack of time to re-evaluate and therefore support a working diagnosis. The building of rapport in non-ESP practice occurred over a period of patient treatments, whereas the ESP typically saw the patient once. Lack of time was a key part of the external/internal pressures facing ESPs and one that ESPs found difficult to deal with;

"And then the time frames of assessing I find stressful" SSI 1409

Having less time meant greater reliance on experience, gutfeeling and fast interpretation.

\section{Discussion}

This research has identified seven themes surrounding clinical reasoning in the assessment of patients reporting low back pain. This analysis provides a novel framework for clinicians, students, educators and health managers that highlights the components of physiotherapists' reasoning and some important differences between ESP and non-ESP practice. Safety and accountability play a relevant part in how the ESP clinician considers the importance of their role and increases these clinicians' levels of anxiety. This particular pressure is not acknowledged by previous clinical reasoning models such as Jones (1995, 1997), Butler (2000); Jones and Rivett (2004). The analysis here also recognises that internal 
and external factors including the clinician's own internal beliefs system have a role in ESP reasoning. The levels of accountability in ESP practice combined with less time to establish a relationship and rapport with the patient added to the stresses associated with this role, and on ESP decision-making. The role of imaging and the shift in physiotherapy practice in line with the extension of roles is highlighted in this research, especially in terms of safety and accountability, and this potentially highlights why gut-feeling and levels of stress seem to be an integral part of practice for ESPs.

In addition, gut-feeling was identified as core to clinicians' reasoning processes. This challenges current physiotherapy models of reasoning. The medical community has provided literature that acknowledges gut-feeling (Stolper et al., 1996), yet physiotherapy appears to retain a residual preference for pattern-recognition as the core process of fast reasoning. Our data suggest that ESP clinicians, while somewhat reticent to recognise gut feeling more publicly agreed that it was "massively important" in their decision making.

There are a number of limitations associated with this study. The reasoning models can only be attributed to the assessment of patients with back pain and therefore are not necessarily transferable to other areas of practice. The focus groups were mixed with ESPs and non-ESPs and so there may have been some reticence from the non-ESPs to offer thoughts in this environment due to a difference in NHS grades. Lastly, it needs to be acknowledged that the role of the researcher in both phases of the study could have possibly influenced the discussions due to a perception by participants of feeling judged when explaining their clinical reasoning.

\section{Conclusions}

Clinical reasoning is a complex and multi-faceted process that appears to have evolved with the extension of roles within physiotherapy. External drivers (such as policy and finance), together with internal pressures related to the clinicians' perceptions of themselves, lack of time, considerations of safety and accountability are all seen to be especially important influences on decision-making by ESPs. In order to deal with these pressures they appear to develop and use gut-feeling to support their clinical reasoning. The use of gut-feeling in this practice, in addition to the stressors facing ESPs as a result of higher perceived levels of accountability and safety, were notable differences as compared to non-ESP physiotherapy clinical reasoning. Further research is now needed to understand gut-feeling better and explore how it might be interpreted to improve clinical reasoning practice.

\section{References}

Arocha JF, Patel VL, Patel YC. Hypothesis generation and the coordination of theory and evidence in novice diagnostic reasoning. Med Decis Mak 1993;13(3): $198-211$.

Banning M. Clinical reasoning and its application to nursing: concepts and research studies. Nurse Educ Pract 2008;8(3):177-83.

Bleakley A, Farrow R, Gould D, Marshall R. Making sense of clinical reasoning: judgement and the evidence of the senses. Med Educ 2003;37(6):544-52.

Butler D,S. The sensitive nervous system. Australia: NOI; 2000.

Charmaz K. Constructing grounded theory. 3rd ed. London: Sage; 2008. p. 42-72. Curran MJ, Campbell J, Rugg G. An investigation into the clinical reasoning of both expert and novice podiatrists. Foot 2006;16(1):28-32.

Daker-White G, Carr AJ, Harvey I, Woolhead G, Bannister G, Nelson I, et al. A randomised controlled trial. Shifting boundaries of doctors and physiotherapists in orthopaedic outpatient departments. J Epidemiol Community Health 1999;53(10):643-50.
Dawson L, Ghazi F. The experience of physiotherapy extended scope practitioners in orthopaedic outpatient clinics. Physiotherapy 2004;90:201-16.

Department of Health. The musculoskeletal services framework. 2006. Available from: www.dh.gov.uk (accessed 11.11.12.).

Department of Health. Patient First and foremost. 2013 (accessed 29.01.14.), www. dh.gov.uk.

Dewar K. Advanced practice and advanced practitioners. In: Clinical judgement and decision-making. England: Open University Press; 2010. p. 28-53.

Doody C, McAteer M. Clinical reasoning of expert and novice physiotherapists in an outpatient setting. Physiotherapy 2002;88(5):258-68.

Edwards I, Jones M, Carr J, Braunack-Mayer A, Jensen GM. Clinical reasoning strategies in physical therapy. Phys Ther 2004;84(4):312-30.

Flynn T, Fritz J, Whitman J, Wainner R, Magel J, Rendeiro D, et al. A clinical prediction rule for classifying patients with low back pain who demonstrate shortterm improvement with spinal manipulation. Spine (Phila Pa 1976) 2002;27(24):2835-43.

Forde R. Competing conceptions of diagnostic reasoning is there a way out? Theor Med Bioeth 1998;19(1):59-72.

Gardiner J, Turner P. Accuracy of clinical diagnosis of internal derangement of the knee by extended scope physiotherapists and orthopaedic doctors: retrospective audit. Physiotherapy 2002;88(3):153-7.

Glaser B, Strauss A. The discovery of grounded theory: strategies for qualitative research. Chicago: Aldine; 1967.

Graves J, Fulton-Kehoe D, Jarvik J, Franklin G. Early imaging for acute low back pain: one-year health and disability outcomes among Washington state workers. Spine 2012a;37(18):1617-27.

Graves J, Fulton-Kehoe D, Martin D, Jarvik J, Franklin G. Factors associated with early magnetic resonance imaging utilisation for acute occupational low back pain: a population-based study from Washington state workers' compensation. Spine 2012b;37(19):1708-18.

Harkapaa K. Relationships of psychological distress and health locus of contro beliefs with the use of cognitive and behavioural coping strategies in low back pain patients. Clin J Pain 1991;7(4):275-82.

Higgs J. Physiotherapy education in the changing international healthcare and educational contexts. Adv Physiother 1999;1(1):17-26.

Higgs J, Jones MA. Clinical decision making and multiple problem spaces. In: Clinical reasoning in the health professions. 3rd ed. London: Elsevier; 2008. p. 3-18.

Jefford E, Fahy K, Sundin D. A review of the literature: midwifery decision-making and birth. Women Birth 2010;23(4):127-34.

Jones MA. Clinical reasoning and pain. Man Ther 1995;1(1):17-24.

Jones MA. Clinical reasoning: the foundation of clinical practice. Part 1. Aust J Physiother 1997;43(3):167-70.

Jones MA, Rivett D. Clinical reasoning for manual therapists. Edinburgh: Butterworth Heineman; 2004.

Leach MJ. Rapport: a key to treatment success. Complement Ther Clin Pract $2005 ; 11(4): 262-5$.

MacNeela P, Gibbons A, McGuire B, Murphy A. We need to get you focused: general practitioners' representations of chronic low back pain patients. Qual Health Res 2010;20(7):977-86.

McPherson K, Kersten P, George S, Lattimer V, Breton A, Ellis B, et al. A systematic review of evidence about extended roles for allied health professionals. J Health Serv Res Policy 2006;11(4):240-7.

May S, Greasley A, Reeve S, Withers S. Expert therapists use specific clinical reasoning processes in the assessment and management of patients with shoulder pain: a qualitative study. Aust J Physiother 2008;54(4):261-6.

Miles M, Huberman A. Qualitative data analysis: an expanded sourcebook. Beverley Hills: Sage; 1994.

Norman G. Research in clinical reasoning: past history and current trends. Med Educ 2005;39(4):418-27.

Patel V, Groen G, Patel Y. Cognitive aspects of clinical performance during patient workup: the role of medical expertise. Adv Health Sci Educ 1997:2:95-114.

Payton OD. Clinical reasoning process in physical therapy. Phys Ther 1985;65(6): 924-8.

Payton OD, Nelson GE, St Clair Hobbs M. Physical therapy patients' perceptions of their relationships with health care professionals. Physiother Theory Pract 1998;14(4):211-21.

Strauss A, Corbin J. Basics of qualitative research: grounded theory procedures and techniques. London: Sage; 1990.

Street JR, Richard L, Makoul G, Arora NK. How does communication heal? Pathways linking clinician-patient communication to health outcomes. Patient Educ Couns 2009;74(3):295-301.

Stolper E, van Royen P, Dinant GJ. The 'sense of alarm' ('gut feeling') in clinical practice. A survey among European general practitioners on recognition and expression. Acute renal infections. Radiol Clin North Am 1996;34(5):965-95.

Videman T, Battie MC, Gibbons LE, Maravilla K, Manninen H, Kaprio J. Associations between back pain history and lumbar MRI findings. Spine 2003;28(6):582-8. 